\title{
A NOVEL WEIGHTING SCHEME FOR RANDOM $k$-SAT
}

\author{
ZONGSHENG GAO, JUN LIU, AND KE XU
}

\begin{abstract}
Consider a random $k$-CNF formula $F_{k}(n, r n)$ with $n$ variables and $r n$ clauses. For every truth assignment $\sigma \in\{0,1\}^{n}$ and every clause $c=\ell_{1} \vee \cdots \vee \ell_{k}$, let $d=d(\sigma, c)$ be the number of satisfied literal occurrences in $c$ under $\sigma$. For fixed $\beta>-1$ and $\lambda>0$, we take

$$
\omega(\sigma, c) \propto\left\{\begin{array}{cl}
0 & d=0 \\
\lambda(1+\beta) & d=1 \\
\lambda^{d} & \text { otherwise. }
\end{array}\right.
$$

Applying the above weighting scheme, we get that if $F_{k}(n, r n)$ is unsatisfiable with probability tending to one as $n \rightarrow \infty$, then $r \geq 2.83,8.09,18.91,40.81$, 84.87 for $k=3,4,5,6$ and 7 , respectively.
\end{abstract}

\section{INTRODUCTION}

Let $V$ be a set of $n$ boolean variables. A $k$-clause is a disjunction of $k$ boolean variables or their negations. Let $C_{k}(V)$ denote the set of all $2^{k} n^{k}$ possible $k$-clauses on $V$. A $k$-CNF formula $F_{k}(n, r n)$ is formed by selecting uniformly, independently and with replacement $r n$ clauses from $C_{k}(V)$ and taking their conjunction [1, 4, 5].

If $k$ is allowed to grow with $n$, Frieze and Wormald [4] proved that if $k-\log _{2} n \rightarrow$ $+\infty$, then random $k$-SAT has a sharp threshold around $n\left(2^{k}+O(1)\right) \ln 2$. A few years later, the authors of this paper [5] relaxed the condition $k-\log _{2} n \rightarrow+\infty$ to $k \geq\left(\frac{1}{2}+\epsilon\right) \log _{2} n$ for any fixed $\epsilon>0$.

For each fixed $k \geq 2$ (i.e., independent of $n$ ), let

$$
r_{k}=\sup \left\{r: \lim _{n \rightarrow \infty} \operatorname{Pr}\left[F_{k}(n, r n) \text { is satisfiable }\right]=1\right\} .
$$

For every truth assignment $\sigma \in\{0,1\}^{n}$ and every clause $c=\ell_{1} \vee \cdots \vee \ell_{k}$, let $d=d(\sigma, c)$ be the number of satisfied literal occurrences in $c$ under $\sigma$. Fix $\lambda>0$ and let

$$
\omega(\sigma, c) \propto\left\{\begin{array}{cl}
0 & d=0, \\
\lambda^{d} & \text { otherwise. }
\end{array}\right.
$$

Applying the above weighting scheme, Achlioptas and Peres [1] proved the following result.

Theorem 1.1. There exists a sequence $\delta_{k} \rightarrow 0$ such that for all $k \geq 3$,

$$
r_{k} \geq 2^{k} \ln 2-(k+1) \frac{\ln 2}{2}-1-\delta_{k} .
$$

2000 Mathematics Subject Classification. Primary 05D40, 60C05; Secondary 68Q25, 82B26.

Key words and phrases. Satisfiability, random formulas, phase transitions, second moment method, weighting scheme.

This research was supported by National Natural Science Fund of China (Grant No.11171013, 60973033). 
This is the first rigorous proof of a replica method prediction for any NP-complete problem at zero temperature, and for $k \geq 4$ this improves all previously known lower bounds for $r_{k}$.

In this paper, we propose a novel weighting scheme, which is a revised version of (1.1)

$$
\omega(\sigma, c) \propto\left\{\begin{array}{cc}
0 & d=0 \\
\lambda(1+\beta) & d=1, \\
\lambda^{d} & \text { otherwise }
\end{array}\right.
$$

where $\beta>-1$ and $\lambda>0$ are fixed.

By choosing $\beta$ and $\lambda$ properly, we will prove that $r_{3} \geq 2.83, r_{4} \geq 8.09, r_{5} \geq 18.91$, $r_{6} \geq 40.81$ and $r_{7} \geq 84.87$ so sharpening the lower bounds $r_{3} \geq 2.68, r_{4} \geq 7.91$, $r_{7} \geq 84.82$ obtained in [1], and $r_{5} \geq 18.79, r_{6} \geq 40.74$ obtained by using the same method as in [1].

\section{The Second Moment Method}

For a non-negative random variable $X$, making use of the second moment $\mathrm{E}\left[X^{2}\right]$ is called the second moment method. In this paper, we use the second moment method in the following form.

Lemma 2.1. For any non-negative random variable $X$,

$$
\operatorname{Pr}[X>0] \geq \frac{\mathbf{E}[X]^{2}}{\mathbf{E}\left[X^{2}\right]}
$$

An attractive feature of the second moment method is that for any non-negative random variable $Y$, if $Y>0$ implies that $X>0$, then

$$
\operatorname{Pr}[X>0] \geq \operatorname{Pr}[Y>0] \geq \frac{\mathbf{E}[Y]^{2}}{\mathbf{E}\left[Y^{2}\right]}
$$

In a breakthrough paper, Friedgut [3] proved the existence of a non-uniform threshold for random $k$-SAT.

Theorem 2.2. For each $k \geq 2$, there exists a sequence $r_{k}(n)$ such that for every $\epsilon>0$,

$$
\lim _{n \rightarrow \infty} \operatorname{Pr}\left[F_{k}(n, r n) \text { is satisfiable }\right]= \begin{cases}1 & r=(1-\epsilon) r_{k}(n), \\ 0 & r=(1+\epsilon) r_{k}(n) .\end{cases}
$$

Given a $k$-CNF formula $\mathrm{F}$ on $n$ variables let $S(F)=\{\sigma: \sigma$ satisfies $F\} \subseteq\{0,1\}^{n}$ denote the set of satisfying truth assignments of $F$ and let $X=X(F) \geq 0$ be such a random variable that $X>0$ implies that $S(F) \neq \emptyset$. Sums of the form

$$
X=\sum_{\sigma} \omega(\sigma, F)
$$

clearly has this property if $\omega(\sigma, F) \geq 0$ and $\omega(\sigma, F)>0$ implies that $\sigma \in S(F)$.

An immediate corollary of Theorem 2.2 is as follows.

Corollary 2.3. For each $k \geq 2$, if $\liminf _{n \rightarrow \infty} \operatorname{Pr}\left[F_{k}(n, r n)\right.$ is satisfiable $]>0$, then $r_{k} \geq r$.

Thus, if for any fixed $r>0$ we have $\mathbf{E}\left[X^{2}\right]=O\left(\mathbf{E}[X]^{2}\right)$, then $r_{k} \geq r$. 
Since $F$ is formed by some independent clauses, it is natural to require that $\omega(\sigma, F)$ has product structure over these clauses

$$
\omega(\sigma, F)=\prod_{c} \omega(\sigma, c),
$$

then clause-independent allows one to replace expectations of products with products of expectations

$$
\mathbf{E}[\omega(\sigma, F)]=\prod_{c} \mathbf{E}[\omega(\sigma, c)]
$$

With this in mind, let us consider random variables of the form

$$
X=\sum_{\sigma} \prod_{c} \omega(\sigma, c),
$$

where $\omega(\sigma, c) \geq 0$ and $\omega(\sigma, c)=0$ if $\sigma$ falsifies $c$.

For every truth assignment $\sigma$ and every clause $c=\ell_{1} \vee \cdots \vee \ell_{k}$, we require that $\omega(\sigma, c)=\omega(\mathbf{v})$, where $\mathbf{v}=\left(v_{1}, \cdots, v_{k}\right), v_{i}=+1$ if $\ell_{i}$ is satisfied under $\sigma$ and -1 if $\ell_{i}$ is falsified under $\sigma$. Since every $\ell_{i}$ in $c$ has the equal right, it is natural to require that $\omega(\mathbf{v})=\omega(|\mathbf{v}|)$, where $|\mathbf{v}|$ denote the number of +1 s in $\mathbf{v}$.

Let $A=\{-1,+1\}^{k}$ and let $\alpha=z / n$. Then [1]

$$
\mathbf{E}[X]=2^{n}\left(\sum_{\mathbf{v} \in A} \omega(\mathbf{v}) 2^{-k}\right)^{r n}, \mathbf{E}\left[X^{2}\right]=2^{n} \sum_{z=0}^{n}\left(\begin{array}{l}
n \\
z
\end{array}\right) f_{\omega}(\alpha)^{r n},
$$

where $f_{\omega}(\alpha)=\sum_{\mathbf{u}, \mathbf{v} \in A} \omega(\mathbf{u}) \omega(\mathbf{v}) 2^{-k} \prod_{i=1}^{k}\left(\alpha^{\mathbf{1}_{u_{i}=v_{i}}}(1-\alpha)^{\mathbf{1}_{u_{i} \neq v_{i}}}\right)$.

The proof of the following Lemma follows by applying the Laplace method of asymptotic analysis [2].

Lemma 2.4. Let $\phi$ be any positive function on $[0,1]$ and let

$$
S_{n}=\sum_{z=0}^{n}\left(\begin{array}{l}
n \\
z
\end{array}\right) \phi(\alpha)^{n}
$$

Letting $0^{0}=1$, define $g$ on $[0,1]$ as

$$
g(\alpha)=\frac{\phi(\alpha)}{\alpha^{\alpha}(1-\alpha)^{1-\alpha}} .
$$

If there exists $\alpha_{\max } \in(0,1)$ such that $g\left(\alpha_{\max }\right) \equiv g_{\max }>g(\alpha)$ for all $\alpha \neq \alpha_{\max }$ and

$$
g^{\prime \prime}\left(\alpha_{\max }\right)=-\frac{g_{\max }}{\alpha_{\max }\left(1-\alpha_{\max }\right)} \times \rho^{-2}\left(\phi \text { is twice differentiable at } \alpha_{\max }\right),
$$

where $\rho>0$, then

$$
\lim _{n \rightarrow \infty} S_{n} / g_{\max }^{n}=\rho
$$

With Lemma 2.4 in mind, let us define

$$
\Lambda_{\omega}(\alpha)=\frac{2 f_{\omega}(\alpha)^{r}}{\alpha^{\alpha}(1-\alpha)^{1-\alpha}}
$$

Observe that

$$
\Lambda_{\omega}(1 / 2)^{n}=\left(4 f_{\omega}(1 / 2)^{r}\right)^{n}=\mathbf{E}[X]^{2} .
$$


Then, Lemma 2.4 and (2.5) can be combined to yield that if $\Lambda_{\omega}$ has a unique global maximum at $1 / 2$ on $[0,1]$ and $\Lambda_{\omega}^{\prime \prime}(1 / 2)<0$, then $\mathbf{E}\left[X^{2}\right]=O\left(\mathbf{E}[X]^{2}\right)$. And we know that [1]

$$
\Lambda_{\omega}^{\prime}(1 / 2)=0 \Longleftrightarrow \sum_{\mathbf{v} \in A} \omega(\mathbf{v})(2|\mathbf{v}|-k)=0 .
$$

The Specific Calculations of Weighting Scheme (1.2). For our weighting scheme, as defined in (1.2), we can rewrite the right side of (2.6) as

$$
\begin{aligned}
& \sum_{j=1}^{k}\left(\begin{array}{c}
k \\
j
\end{array}\right) \lambda^{j}(2 j-k)+k(2-k) \lambda \beta \\
= & k\left(1-(k-2) \lambda \beta-(1+\lambda)^{k-1}(1-\lambda)\right) \\
= & 0,
\end{aligned}
$$

i.e.,

$$
(1+\lambda)^{k-1}(1-\lambda)+(k-2) \lambda \beta=1 .
$$

For every truth assignment $\sigma$ and every clause $c=\ell_{1} \vee \cdots \vee \ell_{k}\left(\ell_{1}, \cdots, \ell_{k}\right.$ are i.i.d.) let $S_{1}(c)=\{\sigma: d(\sigma, c)=1\}$ and let $H(\sigma, c)$ be the number of satisfied literal occurrences in $c$ under $\sigma$ less the number of unsatisfied literal occurrences in $c$ under $\sigma$. For any $\gamma>0$, let

$$
X=\sum_{\sigma} \prod_{c} \gamma^{H(\sigma, c)}\left(\mathbf{1}_{\sigma \in S(c)}+\beta \times \mathbf{1}_{\sigma \in S_{1}(c)}\right) .
$$

(Note that $\gamma^{H(\sigma, c)}=\gamma^{2 d(\sigma, c)-k}$, so this is consistent with (1.2) for $\gamma^{2}=\lambda$.)

Let $\sigma, \tau$ be any pair of truth assignments that agree on $z=\alpha n$ variables. Then

$$
\begin{aligned}
& \mathbf{E}\left[\gamma^{H(\sigma, c)+H(\tau, c)}\right]=\left(\alpha\left(\frac{\gamma^{2}+\gamma^{-2}}{2}\right)+1-\alpha\right)^{k}, \\
& \mathbf{E}\left[\gamma^{H(\sigma, c)+H(\tau, c)} \mathbf{1}_{\sigma \notin S(c)}\right]=\mathrm{E}\left[\gamma^{H(\sigma, c)+H(\tau, c)} \mathbf{1}_{\tau \notin S(c)}\right]=\left(\frac{\alpha \gamma^{-2}+1-\alpha}{2}\right)^{k}, \\
& \mathbf{E}\left[\gamma^{H(\sigma, c)+H(\tau, c)} \mathbf{1}_{\sigma, \tau \notin S(c)}\right]=\left(\frac{\alpha \gamma^{-2}}{2}\right)^{k}, \\
& \mathbf{E}\left[\gamma^{H(\sigma, c)+H(\tau, c)} \mathbf{1}_{\sigma \in S_{1}(c)}\right]=\mathrm{E}\left[\gamma^{H(\sigma, c)+H(\tau, c)} \mathbf{1}_{\tau \in S_{1}(c)}\right] \\
= & \left(\begin{array}{l}
k \\
1
\end{array}\right)\left(\frac{\alpha \gamma^{2}+1-\alpha}{2}\right)\left(\frac{\alpha \gamma^{-2}+1-\alpha}{2}\right)^{k-1}, \\
& \mathbf{E}\left[\gamma^{H(\sigma, c)+H(\tau, c)} \mathbf{1}_{\sigma \notin S(c), \tau \in S_{1}(c)}\right]=\mathrm{E}\left[\gamma^{H(\sigma, c)+H(\tau, c)} \mathbf{1}_{\sigma \in S_{1}(c), \tau \notin S(c)}\right] \\
= & \left(\begin{array}{c}
k \\
1
\end{array}\right)\left(\frac{1-\alpha}{2}\right)\left(\frac{\alpha \gamma^{-2}}{2}\right)^{k-1}, \\
& \mathbf{E}\left[\gamma^{H(\sigma, c)+H(\tau, c)} \mathbf{1}_{\sigma, \tau \in S_{1}(c)}\right] \\
= & \left(\begin{array}{c}
k \\
1
\end{array}\right)\left(\frac{\alpha \gamma^{2}}{2}\right)\left(\frac{\alpha \gamma^{-2}}{2}\right)^{k-1}+2 !\left(\begin{array}{c}
k \\
2
\end{array}\right)\left(\frac{1-\alpha}{2}\right)^{2}\left(\frac{\alpha \gamma^{-2}}{2}\right)^{k-2} .
\end{aligned}
$$


Observe that

$$
\begin{aligned}
& \left(\mathbf{1}_{\sigma \in S(c)}+\beta \times \mathbf{1}_{\sigma \in S_{1}(c)}\right)\left(\mathbf{1}_{\tau \in S(c)}+\beta \times \mathbf{1}_{\tau \in S_{1}(c)}\right) \\
= & \left(\mathbf{1}-\mathbf{1}_{\sigma \notin S(c)}+\beta \times \mathbf{1}_{\sigma \in S_{1}(c)}\right)\left(\mathbf{1}-\mathbf{1}_{\tau \notin S(c)}+\beta \times \mathbf{1}_{\tau \in S_{1}(c)}\right) \\
= & \mathbf{1}-\mathbf{1}_{\sigma \notin S(c)}-\mathbf{1}_{\tau \notin S(c)}+\mathbf{1}_{\sigma, \tau \notin S(c)}+ \\
& \beta \times\left(\mathbf{1}_{\sigma \in S_{1}(c)}+\mathbf{1}_{\tau \in S_{1}(c)}-\mathbf{1}_{\sigma \in S_{1}(c), \tau \notin S(c)}-\mathbf{1}_{\sigma \notin S(c), \tau \in S_{1}(c)}\right)+ \\
& \beta^{2} \times \mathbf{1}_{\sigma, \tau \in S_{1}(c)} \equiv \Gamma((\sigma, \tau), \beta, c) .
\end{aligned}
$$

Therefore

$$
\begin{aligned}
& \mathbf{E}\left[\gamma^{H(\sigma, c)+H(\tau, c)} \Gamma((\sigma, \tau), \beta, c)\right] \\
= & A(\alpha, \gamma)+2 k \times B(\alpha, \gamma) \times \beta+k \times C(\alpha, \gamma) \times \beta^{2} \\
\equiv & f(\alpha, \beta, \gamma),
\end{aligned}
$$

where

$$
\begin{aligned}
& A(\alpha, \gamma)=\left(\alpha\left(\frac{\gamma^{2}+\gamma^{-2}}{2}\right)+1-\alpha\right)^{k}-2\left(\frac{\alpha \gamma^{-2}+1-\alpha}{2}\right)^{k}+\left(\frac{\alpha \gamma^{-2}}{2}\right)^{k}, \\
& B(\alpha, \gamma)=\left(\frac{\alpha \gamma^{2}+1-\alpha}{2}\right)\left(\frac{\alpha \gamma^{-2}+1-\alpha}{2}\right)^{k-1}-\left(\frac{1-\alpha}{2}\right)\left(\frac{\alpha \gamma^{-2}}{2}\right)^{k-1}, \\
& C(\alpha, \gamma)=\left(\frac{\alpha \gamma^{2}}{2}\right)\left(\frac{\alpha \gamma^{-2}}{2}\right)^{k-1}+(k-1)\left(\frac{1-\alpha}{2}\right)^{2}\left(\frac{\alpha \gamma^{-2}}{2}\right)^{k-2} .
\end{aligned}
$$

Then, (2.9) and (2.10) can be combined to yield that

$$
\begin{aligned}
\mathbf{E}\left[X^{2}\right] & =\mathbf{E}\left[\sum_{\sigma, \tau} \prod_{c} \gamma^{H(\sigma, c)+H(\tau, c)} \Gamma((\sigma, \tau), \beta, c)\right] \\
& =\sum_{\sigma, \tau} \prod_{c} \mathbf{E}\left[\gamma^{H(\sigma, c)+H(\tau, c)} \Gamma((\sigma, \tau), \beta, c)\right] \\
& =2^{n} \sum_{z=0}^{n}\left(\begin{array}{l}
n \\
z
\end{array}\right) f(\alpha, \beta, \gamma)^{r n} .
\end{aligned}
$$

With Lemma 2.4 in mind, let us define

$$
G_{r}(\alpha, \beta, \gamma)=\frac{f(\alpha, \beta, \gamma)^{r}}{\alpha^{\alpha}(1-\alpha)^{1-\alpha}}
$$

Fix $\beta$ and $\gamma$. Then, if $G_{r}$ has a unique global maximum at $\alpha=1 / 2$ on $[0,1]$ and

$$
\frac{\partial^{2} G_{r}}{\partial \alpha^{2}}(1 / 2, \beta, \gamma)<0
$$

then we get $r_{k} \geq r$.

An Enhanced Method: Truncation and Weighting. For a given $k$-CNF formula $F$ on $n$ variables, recall that $S=S(F) \subseteq\{0,1\}^{n}$ is the set of satisfying truth assignment of $F$. Let $S^{+}=\{\sigma \in S: H(\sigma, F) \geq 0\}$ and let $X_{+}=$ $\sum_{\sigma \in S^{+}} \prod_{c} \omega(\sigma, c)$. For any weighting scheme of $k$-SAT: $\omega(\sigma, c) \geq 0$ and $\omega(\sigma, c)=0$ if $\sigma$ falsifies $c$, we have [6] 
Lemma 2.5. If $f_{\omega}^{\prime}(1 / 2)=0$, then $\lim _{n \rightarrow \infty} \mathbf{E}\left[X_{+}\right] / \mathbf{E}[X]=1 / 2$.

For any $\gamma>0$, let

$$
X_{+}=\sum_{\sigma \in S^{+}} \prod_{c} \gamma^{H(\sigma, c)}\left(\mathbf{1}_{\sigma \in S(c)}+\beta \times \mathbf{1}_{\sigma \in S_{1}(c)}\right) .
$$

(This is consistent with those random variables $X$ defined in (2.8), i.e., consistent with our weighting scheme (1.2).)

Observe that

$$
f_{\omega}^{\prime}(1 / 2)=0 \Longleftrightarrow\left(1+\gamma^{2}\right)^{k-1}\left(1-\gamma^{2}\right)+(k-2) \gamma^{2} \beta=1 .
$$

A simple calculation gives

$$
\begin{aligned}
X_{+}^{2} & =\left(\sum_{\sigma \in S^{+}} \prod_{c} \omega(\sigma, c)\right)\left(\sum_{\tau \in S^{+}} \prod_{c} \omega(\tau, c)\right) \\
& =\left(\sum_{\sigma} \mathbf{1}_{\sigma \in S^{+}} \prod_{c} \omega(\sigma, c)\right)\left(\sum_{\tau} \mathbf{1}_{\tau \in S^{+}} \prod_{c} \omega(\tau, c)\right) \\
& =\sum_{\sigma, \tau} \mathbf{1}_{\sigma, \tau \in S^{+}} \prod_{c} \omega(\sigma, c) \omega(\tau, c) \\
& =\sum_{\sigma, \tau} \mathbf{1}_{\sigma, \tau \in S^{+}} \prod_{c} \gamma^{H(\sigma, c)+H(\tau, c)} \Gamma((\sigma, \tau), \beta, c) .
\end{aligned}
$$

Given a tuple $\left(\beta_{0}, \gamma_{0}\right) \in(-1,+\infty) \times(0,+\infty)$ satisfies the right side of (2.13). In particular, if

$$
X_{+}=\sum_{\sigma \in S^{+}} \prod_{c} \gamma_{0}^{H(\sigma, c)}\left(\mathbf{1}_{\sigma \in S(c)}+\beta_{0} \times \mathbf{1}_{\sigma \in S_{1}(c)}\right),
$$

then for any $\gamma \geq \gamma_{0}$, following the derivation of (2.11) and (2.14), we deduce that

$$
\begin{aligned}
\mathbf{E}\left[X_{+}^{2}\right] & =\sum_{\sigma, \tau} \mathbf{E}\left[\mathbf{1}_{\sigma, \tau \in S^{+}} \prod_{c} \gamma_{0}^{H(\sigma, c)+H(\tau, c)} \Gamma\left((\sigma, \tau), \beta_{0}, c\right)\right] \\
& \leq \sum_{\sigma, \tau} \mathbf{E}\left[\prod_{c} \gamma^{H(\sigma, c)+H(\tau, c)} \Gamma\left((\sigma, \tau), \beta_{0}, c\right)\right] \\
& =\sum_{\sigma, \tau} \mathbf{E}\left[\gamma^{H(\sigma, c)+H(\tau, c)} \Gamma\left((\sigma, \tau), \beta_{0}, c\right)\right]^{r n} \\
& =2^{n} \sum_{z=0}^{n}\left(\begin{array}{l}
n \\
z
\end{array}\right) f\left(\alpha, \beta_{0}, \gamma\right)^{r n} .
\end{aligned}
$$

Therefore

$$
\mathbf{E}\left[X_{+}^{2}\right] \leq 2^{n} \sum_{z=0}^{n}\left(\begin{array}{l}
n \\
z
\end{array}\right) \inf _{\gamma \geq \gamma_{0}} f\left(\alpha, \beta_{0}, \gamma\right)^{r n} \equiv 2^{n} \sum_{z=0}^{n}\left(\begin{array}{l}
n \\
z
\end{array}\right) \underline{f}\left(\alpha, \beta_{0}, \gamma_{0}\right)^{r n} .
$$

With Lemma 2.4 in mind and we take

$$
g_{r}\left(\alpha, \beta_{0}, \gamma_{0}\right)=\frac{\underline{f}\left(\alpha, \beta_{0}, \gamma_{0}\right)^{r}}{\alpha^{\alpha}(1-\alpha)^{1-\alpha}}\left(\leq \frac{f\left(\alpha, \beta_{0}, \gamma_{0}\right)^{r}}{\alpha^{\alpha}(1-\alpha)^{1-\alpha}}=G_{r}\left(\alpha, \beta_{0}, \gamma_{0}\right)\right) .
$$

Note now that if $g_{r}\left(1 / 2, \beta_{0}, \gamma_{0}\right)>g_{r}\left(\alpha, \beta_{0}, \gamma_{0}\right)$ for all $\alpha \neq 1 / 2$, then

$$
g_{r}\left(1 / 2, \beta_{0}, \gamma_{0}\right)=G_{r}\left(1 / 2, \beta_{0}, \gamma_{0}\right),
$$


otherwise, let $\varepsilon=G_{r}\left(1 / 2, \beta_{0}, \gamma_{0}\right)-g_{r}\left(1 / 2, \beta_{0}, \gamma_{0}\right)(>0)$, then there exists a constant $D>0$ such that for all sufficiently large $n$,

$$
\begin{aligned}
\mathbf{E}\left[X_{+}^{2}\right] & \leq\left(2 g_{r}\left(1 / 2, \beta_{0}, \gamma_{0}\right)+\varepsilon\right)^{n} \leq\left(2 g_{r}\left(1 / 2, \beta_{0}, \gamma_{0}\right)+2 \varepsilon\right)^{n} \\
& =\left(2 G_{r}\left(1 / 2, \beta_{0}, \gamma_{0}\right)\right)^{n}=\mathbf{E}[X]^{2} \leq D \times \mathbf{E}\left[X_{+}\right]^{2} \\
& \leq D \times \mathbf{E}\left[X_{+}^{2}\right] .
\end{aligned}
$$

Note that

$$
\left(\frac{2 g_{r}\left(1 / 2, \beta_{0}, \gamma_{0}\right)+2 \varepsilon}{2 g_{r}\left(1 / 2, \beta_{0}, \gamma_{0}\right)+\varepsilon}\right)^{n} \rightarrow+\infty \text { as } n \rightarrow \infty
$$

and this is a contradiction.

Suppose that $g_{r}\left(1 / 2, \beta_{0}, \gamma_{0}\right)>g_{r}\left(\alpha, \beta_{0}, \gamma_{0}\right)$ for all $\alpha \neq 1 / 2$ and

$$
\frac{\partial^{2} G_{r}}{\partial \alpha^{2}}\left(1 / 2, \beta_{0}, \gamma_{0}\right)<0 \text {. }
$$

Note that there exists a constant $\epsilon$ such that $G_{r}\left(1 / 2, \beta_{0}, \gamma_{0}\right)>G_{r}\left(\alpha, \beta_{0}, \gamma_{0}\right)$ hold on for all $\alpha \in(1 / 2-\epsilon, 1 / 2) \cup(1 / 2,1 / 2+\epsilon)$. With Lemma 2.4 in mind, we consider the function defined as follows:

$$
\phi\left(\alpha, \beta_{0}, \gamma_{0}\right)= \begin{cases}f\left(\alpha, \beta_{0}, \gamma_{0}\right) & \text { if } \alpha \in(1 / 2-\epsilon, 1 / 2+\epsilon), \\ \underline{f}\left(\alpha, \beta_{0}, \gamma_{0}\right) & \text { otherwise. }\end{cases}
$$

It is clear that

$$
\mathbf{E}\left[X_{+}^{2}\right] \leq \sum_{z=0}^{n}\left(\begin{array}{l}
n \\
z
\end{array}\right) \phi\left(\alpha, \beta_{0}, \gamma_{0}\right)^{r n}
$$

then by Lemma 2.4, we get $r_{k} \geq r$.

\section{Use of the Method}

We apply the method to the case $k=3,4,5,6$ and 7 . To do this, we demonstrate values $\beta, r$ and let $\gamma$ satisfies the right side of (2.13) such that $g_{r}(1 / 2, \beta, \gamma)>$ $g_{r}(\alpha, \beta, \gamma)$ hold on for all $\alpha \neq 1 / 2$ and

We obtain

$$
\frac{\partial^{2} G_{r}}{\partial \alpha^{2}}(1 / 2, \beta, \gamma)<0
$$

\begin{tabular}{cccccc}
$k$ & 3 & 4 & 5 & 6 & 7 \\
\hline$\beta$ & $(0.56,0.74)$ & $(0.13,0.15)$ & $(0.04,0.06)$ & 0.02 & 0.01 \\
$r$ & 2.83 & 8.09 & 18.91 & 40.81 & 84.87
\end{tabular}

\section{REFERENCES}

[1] D. Achlioptas and Y. Peres: The threshold for random $k$-SAT is $2^{k} \log 2-O(k)$, J. Amer. Math. Soc. 17 (2004), 947-973.

[2] N. G. De Bruijn: Asymptotic methods in analysis, 3rd ed., Dover Publications Inc., New York, 1981.

[3] E. FRIEdGut: Necessary and sufficient conditions for sharp thresholds of graph properties, and the k-SAT problem, J. Amer. Math. Soc. 12 (1999), 1017-1054.

[4] A. Frieze and N. C. Wormald: Random k-SAT: A tight threshold for moderately growing $k$, Combinatorica 25 (2005), 297-305.

[5] J. Liu, Z. GaO and K. Xu: A Note on Random k-SAT for Moderately Growing k, E-JC 19 (2012), \#P24.

[6] F. Yu. Vorobyev: A lower bound for the 4-satisfiability threshold, Discrete Math. Appl. 17(3) (2007), 287-294. 
Zongsheng Gao: LmiB and School of Mathematics and Systems Science, Beihang University, Beijing, 100191, P.R. China

E-mail address: zshgao@buaa.edu.cn

Jun Liu: LmiB and School of Mathematics and Systems Science, Beinang University, BeIJing, 100191, P.R. China

E-mail address: junliu@smss.buaa.edu.cn

Ke Xu: State Key Laboratory of Software Development Environment, Department of Computer Science, Beinang University, Beijing, 100191, P.R. China

E-mail address: kexu@nlsde.buaa.edu.cn 